\title{
LA COMPOSICIÓN CORPORAL COMO MEDICIÓN INDIRECTA DE LA ACTIVIDAD FÍSICA Y SU RELACION CON EL RENDIMIENTO ACADÉMICO EN ESTUDIANTES DEL GRADO DE EDUCACIÓN INFANTIL
}

\author{
Amara García Belloso \\ Universidad de Extremadura \\ amgarciab@alumnos.unex.es \\ Ángel Ventura García Preciado \\ Universidad de Extremadura \\ vengar@unex.es
}

Recepción Artículo: 18 septiembre 2021 Admisión Evaluación: 18 septiembre 2021 Informe Evaluador 1: 26 septiembre 2021 Informe Evaluador 2: 27 septiembre 2021 Aprobación Publicación: 29 septiembre 2021

\section{RESUMEN}

Desde la antigüedad se conocen múltiples beneficios de la actividad física. A día de hoy, se han realizado múltiples estudios que analizan la asociación entre los niveles de actividad física y la evaluación del rendimiento académico, encontrándose diversas contradicciones. Por esta razón, se pretende conocer indirectamente el nivel de actividad física y hábitos saludables mediante el análisis de la constitución del cuerpo, es decir, de la composición corporal para ver la relación entre sexos y rendimiento académico. Se ha realizado una metodología de investigación cuantitativa mediante el método descriptivo en un aula del tercer curso de Educación Infantil de la Universidad de Extremadura. El tamaño de la muestra es de 61 participantes, con edades entre 20-28 años, de Ios cuales, 48 son de género femenino y 13 de género masculino. Como instrumento de recogida de datos se ha utilizado una báscula de bioimpedancia, recogiendo los datos manualmente sobre una plantilla y posteriormente a una hoja de cálculo Excel. Se ha realizado un análisis profundo prestando voz a los resultados extraídos mediante tablas y gráficos. Según los resultados de estudio, se ha detectado niveles saludables de peso, porcentaje de masa muscular, densidad de masa ósea, IMC en mujeres (73\%), porcentaje agua corporal y grasa visceral. En cambio, el porcentaje de masa corporal, el IMC en hombres (54\%) y la edad metabólica indican niveles negativos o no saludables. A modo de conclusión, se afirma que los estudiantes de $3^{0}$ del grado de Educación Infantil presentan un buen estado de condición física y de salud, de manera que, se llega a la conclusión de que realizan un nivel adecuado de actividad física. Sin embargo, todos presentan buenos resultados académicos, presentando poca variabilidad entre las notas, por este motivo, no se han podido sacar conclusiones claras sobre la actividad física en relación al rendimiento académico.

Palabras clave: metodología de investigación (520201); niveles de actividad (610605); evaluación del rendimiento (610907); constitución del cuerpo (240205) 


\title{
LA COMPOSICIÓN CORPORAL COMO MEDICIÓN INDIRECTA DE LA ACTIVIDAD FÍSICA Y SU RELACIÓN CON EL RENDIMIENTO ACADÉMICO EN ESTUDIANTES DEL GRADO DE EDUCACIÓN INFANTIL
}

\begin{abstract}
Body composition as an indirect measure of physical activity and its relationship with academic performance in early childhood education students. Multiple benefits of physical activity have been known since ancient times. To date, there have been multiple studies that analyze the association between levels of physical activity and the evaluation of academic performance, finding various contradictions. For this reason, it is intended to indirectly know the level of physical activity and healthy habits by analyzing the constitution of the body, that is, of the body composition to see the relationship between sex and academic performance. A quantitative research methodology has been carried out using the descriptive method in a classroom of the third year of Early Childhood Education at the University of Extremadura. The sample size is 61 participants, aged 2028 years, of which 48 are female and 13 are male. As a data collection instrument, a bioimpedance scale has been used, collecting the data manually on a template and later on an Excel spreadsheet. An in-depth analysis has been carried out giving voice to the results extracted through tables and graphs. According to the study results, healthy levels of weight, muscle mass percentage, bone mass density, BMI in women (73\%), body water percentage and visceral fat have been detected. On the other hand, the percentage of body mass, the BMI in men (54\%) and the metabolic age indicate negative or unhealthy levels. By way of conclusion, it is stated that the students in the 3rd grade of Early Childhood Education present a good state of physical condition and health, so that it is concluded that they carry out an adequate level of physical activity. However, they all present good academic results, presenting little variability between grades, for this reason, it has not been possible to draw clear conclusions about physical activity in relation to academic performance.
\end{abstract}

Keywords: research methodology (520201); activity levels (610605); performance evaluation (610907); body constitution (240205)

\section{INTRODUCCIÓN}

Desde la antigüedad se conocen múltiples beneficios que la actividad física (AF) aporta al ser humano (Alfaro 2018:23). Gracias a la neurobiología, se sabe que a través de la AF se mantiene aportes necesarios de nutrientes al cerebro y optimiza la eficacia funcional de las redes neuronales, alcanzando un mejor desarrollo y estimulo entre sus conexiones, favoreciendo la neurogénesis. Así pues, el cuerpo necesita la AF para mantener una serie de funciones básicas (Barrios y López, 2016). Asimismo, la AF está vinculada a los procesos neurocognitivos en las diferentes etapas del desarrollo humano, donde favorece neuroplasticidad en las edades más tempranas (Cortés, Veloso y Alfaro 2020:40) y disminuye el desarrollo de enfermedades neurodegenerativas en edades más avanzadas (Ponce, 2015). De manera que, el desarrollo del sistema nervioso central humano es modulado por la actividad física (Cortés, Veloso y Alfaro 2020) porque en él, actúan diversas proteínas como la BDNF (factor neurotrófico derivado del cerebro), una proteína de factores de crecimiento que promueve el desarrollo de neuronas inmaduras ayudando a su supervivencia, así como, la formación de la memoria, el aprendizaje, la plasticidad, la sináptica, la conectividad neuronal... y su síntesis es estimulada por la práctica de ejercicio físico (Cid, 2016).

Debido a estas afirmaciones, diversos estudios determinan la incidencia de la AF en el rendimiento académico (RA), siendo un creciente objeto de investigación en niños, adolescentes y universitarios. Muchos algunos autores focalizan las investigaciones en personas que estudian en niveles superiores, debido a que los universitarios a menudo sufren problemas de estrés, pudiendo afectar a su rendimiento académico.

Por otro lado, la Actividad física (AF) está interrelacionada con la condición física (CF) y ésta, a su vez, con la condición corporal (CC). Para evaluar la CC se tiene en cuenta diferentes medidas como: el peso, el porcentaje de grasa corporal, la masa muscular, la masa ósea, el índice de masa corporal (IMC), la edad metabólica, el porcentaje de agua total del cuerpo y el nivel de grasa visceral. Pero los métodos que miden de forma precisa la CC, son a menudo muy costosos y poco accesibles (Lara et. al, 2014). No obstante, los problemas actuales de la sociedad y los avances tecnológicos han fomentado el desarrollo de numerosas técnicas para su medición, como las técnicas doblemente indirectas, por ejemplo, la bioimpedancia.

Actualmente se encuentran diversos dispositivos que utilizan el método de impedancia bioeléctrica (BIA) 
para medir la CC, por ejemplo, las básculas de bioimpedancia (Santos, 2011) de forma accesible y fiable. Debido al descubrimiento de nuevas herramientas que permite estimar la actividad física de una persona, se plantea la siguiente pregunta de investigación: ¿Existe relación entre actividad física y rendimiento académico, estimando el nivel de actividad física de una persona mediante el estudio de su composición corporal? Por esta razón, se pretende conocer indirectamente el nivel de actividad física y hábitos saludables mediante el análisis de la composición corporal, para ver la relación entre sexos y rendimiento académico.

\section{Objetivos}

Así pues, el objetivo que persigue esta investigación es: Conocer indirectamente el nivel de actividad física y hábitos saludables mediante el análisis de la composición corporal de los estudiantes del aula de $3^{0}$ del grado en Educación Infantil, para ver la relación entre sexos y rendimiento académico. Por consiguiente, se plantean los siguientes objetivos específicos de la investigación: Realizar un análisis descriptivo de cada elemento de composición corporal según el sexo y según el rendimiento académico; y sacar conclusiones en cuanto a la relación de actividad física y rendimiento académico. Además, las variables que se van a controlar son: el nivel de actividad física y factores de la composición corporal medidas a través de la bioimpedancia: peso, porcentaje de grasa corporal, porcentaje de masa muscular, densidad ósea, índice de masa corporal, porcentaje de agua corporal, proporción de grasa visceral, edad metabólica como variable dependiente; y el rendimiento académico y sexo como variable independiente.

\section{MÉTODO}

Este estudio, realiza una investigación cuantitativa mediante el método descriptivo sobre una población determinada en relación con las variables a estudiar.

\section{Población y Muestra}

La población escogida para el estudio es el aula de Expresión, comunicación y creación en las manifestaciones motrices del turno de mañana del $3^{0}$ curso del grado de Educación Infantil, compuesta por 80 alumnos. Debido a dificultades de acceso para trabajar con toda la población, se ha decidido que el tamaño muestral será de 61 participantes. Para ello, se ha seleccionado a la muestra mediante un muestreo aleatorio simple, de tal forma que todas las muestras posibles tienen la misma probabilidad de ser elegidas y así, se podrá realizar estimaciones en cuanto a la población escogida. Gracias a Excel, se ha realizado el método de muestreo aleatorio, obteniendo un total de 61 estudiantes del conjunto de la población (80).

Teniendo en cuenta que se han escogido 61 (n) participantes de $80(\mathrm{~N})$ en total, se ha decidido un nivel de confianza 85\%, que corresponde a una puntuación z de 1,6 (z), por lo que se presentaría un margen de error de $0,05(5 \%)$. Esta medida indica la medida que se espera que los resultados puedan reflejarse a la población en general, es decir, el rango de los valores que se encuentran por encima y por debajo de los resultados reales encontrados. Los 61 participantes presentan con un rango de edad entre 20-28 años, de los cuales, 48 son mujeres y 13 son hombres. Cabe destacar que la media y la moda de edad es de 21 años.

Los criterios de exclusión para la realización del estudio fueron embarazadas, los elementos metálicos en el cuerpo o personas con dispositivo médico electrónico implantado, tales como un marca pasos, debido a la interferencia con el funcionamiento del dispositivo. Sin embargo, ninguno se encontraba en dichas situaciones.

\section{Instrumento de Recogida de Datos y Variables de Estudio}

Se ha utilizado el método de BIA para la medición de la composición corporal de todos los participantes al comienzo del experimento con la báscula de bioimpedancia Tanita BC-601F ES. Este monitor presenta cuatro electrodos, dos para los pies y dos para las manos. Asimismo, permite realizar lecturas sobre la CC, tales como el peso, el porcentaje de grasa corporal, la masa muscular, la masa ósea, el índice de masa corporal (BMI), la ingestión diaria de calorías (DCI), la edad metabólica, el porcentaje de agua total del cuerpo y el nivel de grasa 


\title{
LA COMPOSICIÓN CORPORAL COMO MEDICIÓN INDIRECTA DE LA ACTIVIDAD FÍSICA Y SU RELACIÓN CON EL RENDIMIENTO ACADÉMICO EN ESTUDIANTES DEL GRADO DE EDUCACIÓN INFANTIL
}

visceral. Por otro lado, para obtener la última nota media de los resultados académicos de los participantes, el profesorado facilitado el acceso a la información a través del DNI de los participantes para un mayor anonimato. Se incorpora en una plantilla los datos de la CC y del RA elaborada previamente para su posterior traslado a una tabla Excel.

\section{PROCEDIMIENTO}

En primer lugar, dentro del aula correspondiente, se explica los objetivos de la investigación y el procedimiento a seguir, advirtiendo de que se realizará de forma anónima y que los datos recogidos se usarán con fines científicos. El investigador llama a los estudiantes seleccionados de dos en dos, para la recogida de sus datos con su consentimiento y cumpliendo los criterios de exclusión anteriormente mencionados.

En segundo lugar, se coloca la báscula en una superficie dura y lisa. Como medida de prevención y optimización del dispositivo, se pasa un trapo humedecido con alcohol en la superficie de la báscula tras cada participante.

En tercer lugar, el investigador programa la báscula en modo invitado para ser utilizado una única vez por cada alumno. Se pulsa "GUEST" y después del pitido deben agarrar con los brazos rectos hacia abajo los electrodos de mano y deben subirse a la báscula descalzos, con los pies limpios y sin exceso de ropa (deben quitarse los abrigos y chaquetas). Después de la medición se registra los primeros datos en una plantilla y en la báscula utilizando el botón "SET" especificando la edad, el sexo, la altura y el número de actividad (1= poco o ningún ejercicio/ 2=ejercicio de poca intensidad en ocasiones/ $3=$ aproximadamente 10 horas a la semana). Posteriormente, se muestra las lecturas que realiza la báscula, las cuales deberán ser recogidas rápidamente en la plantilla por el investigador o los voluntarios: peso; porcentaje de grasa corporal; masa muscular; masa ósea; índice de masa corporal (IMC); edad metabólica; porcentaje de agua total del cuerpo; nivel de grasa visceral. Este proceso dura aproximadamente 4 minutos por grupo.

Una vez recogido los datos personales y los datos que proporciona la báscula, se anota la última nota media de los resultados académicos de los participantes, insertando el DNI para un mayor anonimato, registrándola también en la plantilla elaborada. Después, se traslada a una hoja de cálculo de Excel, similar a la plantilla donde se ha recogido los datos.

Finalmente, se realiza un análisis descriptivo, a través de cuadros y gráficas, donde se puede observar la correspondencia entre variables.

\begin{abstract}
Análisis
Se estudia la correlación entre las variables a través del programa Excel. Para ello, se realiza un análisis descriptivo mediante gráficos y tablas.

En primer lugar, se pasan las variables continuas a variables discretas en cinco intervalos, diferenciándolas según el sexo, para conocer la distribución estadística vinculada a una variable, comprendida en intervalos acorde a la Campana de Gauss. Para ello, en cada variable continua se ha seguido los siguientes paso: a) Se ha hallado la media, la desviación típica, la máxima y la mínima en ambos sexos; b) Se ha averiguando la amplitud del intervalo, restando el dato máximo con el mínimo; c) se ha obtenido los valores extremos de cada intervalo dividiendo el resultado entre cinco; d) se ha sumado el intervalo a la mínima hasta llegar a la máxima; e) se ha aplicado la función SI(prueba_lógica1;"valor si verdadero1";SI(prueba_lógica2;"valor si verdadero2",...," con valores del 1 al 5 en una columna auxiliar a la variable continua para incluirla en su intervalo correspondiente. Estos pasos no se han realizado en las siguientes variables: el IMC, ya que cuenta con el sexo, donde se ha utilizado Ios criterios SEEDO que proporciona la sociedad Española de Obesidad (2020) para crear los 6 intervalos; la edad metabólica, donde se ha comprendido en 9 intervalos; el porcentaje de grasa visceral, diferenciándolo entre los parámetros considerados saludables (1-12) y peligrosos (12-59); y el nivel de actividad física, comprendido en 3 intervalos, según la respuesta de los participantes.
\end{abstract}


En segundo lugar se han elaborado unas tablas dinámicas con cada variable dependiente (elementos de la composición corporal), en relación con las variables independiente (sexo y rendimiento académico). Se han añadido los valores relativos a cada valor absoluto para comparar los distintos resultados. Al no usar pruebas probabilísticas para el muestreo, no se puede determinar el margen de error.

Finalmente, se realiza una interpretación de los datos prestando voz a los mismos, acompañado de elementos visuales como gráficas para observar de forma clara los resultados más significativos.

\section{RESULTADOS}

A continuación, se muestran los resultados de los elementos de la composición corporal que se relacionan de forma indirecta con el nivel de actividad física y hábitos saludables, para comprobar su relación entre el sexo y el rendimiento académico, acompañado de gráficas para ver en qué intervalo se presenta el mejor rendimiento.

También se muestran los resultados de la respuesta a una sencilla pregunta que se formuló a los sujetos en relación a su nivel de actividad física, siendo ésta una medición directa para observar la relación entre la variable de sexo y de rendimiento académico, y así, poder sacar conclusiones finales.

\section{Peso}

Teniendo en cuenta que no un existe un peso ideal sin considerar antes otros aspectos como la altura, se ha dividido la muestra en 5 intervalos, desde menor valor hasta el mayor, como forma de descripción de los participantes. No obstante, se puede valorar que los rangos mínimos, bajos e intermedios son saludables y que el intervalo alto y máximo se valora como no saludable.

Tabla 1.

Variable peso en relación al sexo y al $R A$.

\begin{tabular}{|c|c|c|c|c|c|c|c|c|c|c|c|c|c|c|c|c|c|}
\hline & & & & \multicolumn{4}{|c|}{ SEXO } & \multicolumn{8}{|c|}{ RENDIMIENTO ACADÉMICO } & & \\
\hline & \multicolumn{3}{|c|}{ Tabla 2: Peso } & \multicolumn{2}{|c|}{ Femenino } & \multicolumn{2}{|c|}{ Masculino } & \multicolumn{2}{|c|}{ 6-7. } & \multicolumn{2}{|c|}{$7-8$} & \multicolumn{2}{|c|}{ 8-9. } & \multicolumn{2}{|c|}{$9-10}$. & \multicolumn{2}{|c|}{ TOTAL } \\
\hline & & q & $\hat{\sigma}$ & $\mathrm{N}$ & $\%$ & $\mathrm{~N}$ & $\%$ & $\mathrm{~N}$ & $\%$ & $\mathrm{~N}$ & $\%$ & $\mathrm{~N}$ & $\%$ & $\mathrm{~N}$ & $\%$ & $\mathrm{~N}$ & $\%$ \\
\hline \multirow{3}{*}{+} & 1. Mínimo & $<52,04$ & $<69,58$ & 9 & 19 & 2 & 15 & 1 & 13 & 6 & 17 & 3 & 20 & 1 & 50 & 11 & 18 \\
\hline & 2. Bajo & \begin{tabular}{|l|}
$52,05-63,78$ \\
\end{tabular} & 69,59-81,56 & 20 & 42 & 5 & 38 & 4 & 50 & 15 & 42 & 6 & 40 & 0 & 0 & 25 & 41 \\
\hline & 3. Intermedio & \begin{tabular}{|l|}
$63,79-75,52$ \\
\end{tabular} & $81,57-93,54$ & 14 & 29 & 4 & 31 & 3 & 38 & 10 & 28 & 4 & 27 & 1 & 50 & 18 & 30 \\
\hline \multirow{3}{*}{-} & 4. Alto & 75,53-87,26 & $93,55-105,52$ & 4 & 8 & 1 & 8 & 0 & 0 & 5 & 14 & 0 & 0 & 0 & 0 & 5 & 8 \\
\hline & 5. Máximo & $>87,27$ & $>105,53$ & 1 & 2 & 1 & 8 & 0 & 0 & 0 & 0 & 2 & 13 & 0 & 0 & 2 & 3 \\
\hline & \multicolumn{3}{|c|}{ Total general } & 48 & 100 & 13 & 100 & 8 & 100 & 36 & 100 & 15 & 100 & 2 & 100 & 61 & 100 \\
\hline
\end{tabular}

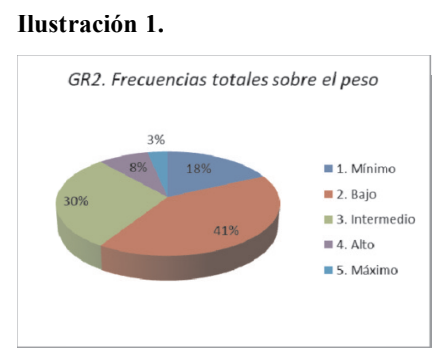

\section{Fuente: elaboración propia.}

Por lo tanto, como se puede apreciar en la ilustración 2, el 41\% de los estudiantes presenta un peso bajo (52-64 en mujeres y 70-82 en hombres) en relación al total de la muestra; el 30\% tienen el peso intermedio; el 18\% el peso mínimo; el 8\% presentan un peso alto; y el 3\% el máximo. Si agrupamos los valores en positivos y negativos, se observa que un $89 \%$ de los estudiantes presenta un peso saludable, mientras que un $10 \%$ tiene un peso no tan saludable. 


\section{LA COMPOSICIÓN CORPORAL COMO MEDICIÓN INDIRECTA DE LA ACTIVIDAD FÍSICA Y SU RELACIÓN CON EL RENDIMIENTO ACADÉMICO EN ESTUDIANTES DEL GRADO DE EDUCACIÓN INFANTIL}

Del mismo modo, en la tabla 2 se muestra la correlación de los datos en relación a dos variables independientes. Respecto al sexo, se observa que el $90 \%$ de las mujeres presenta un peso positivo (mínimo, bajo e intermedio), mientras que tan sólo 5 alumnas, es decir el 10\% las de éstas, presenta un peso negativo (intervalo alto y máximo). Valores similares son los que presentan los hombres, $84 \%$ con valores positivos y $16 \%$ valores negativos de peso, valores muy próximos a la media total.

En cuanto al rendimiento académico, si agrupamos los datos, observamos que el $65 \%$ de la muestra con un peso saludable, presenta un rendimiento académico bueno (notas comprendidas entre 6 y 8), mientras que un $25 \%$ presentan notas superiores (entre 8 y 10). A diferencia de estos, aquellos alumnos con un valor negativo en cuanto al peso, un $8 \%$ presenta un rendimiento académico entre 6-8 y tan solo un 3\% un rendimiento académico de excelente (8-10).

\section{Porcentaje de Grasa Corporal}

Según los criterios de adiposidad que ofrece Dávila-Batista, existe unos porcentajes de grasa corporal saludables, donde en mujeres comprende entre 20 y 30 y en hombres entre 10-20, por lo que los dos primeros intervalos son considerados positivos y los tres últimos negativos.

Tabla 2.

Variable grasa corporal en relación al sexo y al RA.

\begin{tabular}{|c|c|c|c|c|c|c|c|c|c|c|c|c|c|c|c|c|c|}
\hline & \multirow{2}{*}{\multicolumn{3}{|c|}{ Tabla 3: Grasa Corporal }} & \multicolumn{4}{|c|}{ SEXO } & \multicolumn{8}{|c|}{ RENDIMIENTO ACADÉMICO } & & \\
\hline & & & & Fem & nino & \multicolumn{2}{|c|}{ Masculino } & \multicolumn{2}{|c|}{$6-7}$. & \multicolumn{2}{|c|}{ 7-8. } & \multicolumn{2}{|c|}{$8-9}$. & \multicolumn{2}{|c|}{ 9-10. } & \multicolumn{2}{|c|}{ TOTAL } \\
\hline & & q & $\delta$ & $\mathrm{N}$ & $\%$ & $\mathrm{~N}$ & $\%$ & $\mathrm{~N}$ & $\%$ & $\mathrm{~N}$ & $\%$ & $\mathrm{~N}$ & $\%$ & $\mathrm{~N}$ & $\%$ & $\mathrm{~N}$ & $\%$ \\
\hline \multirow{2}{*}{+} & 1. Mínima & $<24,2$ & $<16,06$ & 11 & 23 & 2 & 15 & 1 & 13 & 9 & 25 & 2 & 13 & 1 & 50 & 13 & 21 \\
\hline & 2. Baja & $24,3-30,4$ & $16,07-21,02$ & 13 & 27 & 4 & 31 & 2 & 25 & 10 & 28 & 5 & 33 & 0 & 0 & 17 & 28 \\
\hline & 3. Intermedia & $30,5-36,6$ & $21,03-25,98$ & 10 & 21 & 3 & 23 & 2 & 25 & 8 & 22 & 3 & 20 & 0 & 0 & 13 & 21 \\
\hline & 4. Alta & $36,7-42,8$ & $25,99-30,94$ & 11 & 23 & 3 & 23 & 3 & 38 & 7 & 19 & 3 & 20 & 1 & 50 & 14 & 23 \\
\hline & 5. Máxima & $>42,9$ & $>30,95$ & 3 & 6 & 1 & 8 & 0 & 0 & 2 & 6 & 2 & 13 & 0 & 0 & 4 & 7 \\
\hline & \multicolumn{3}{|c|}{ Total general } & 48 & 100 & 13 & 100 & 8 & 100 & 36 & 100 & 15 & 100 & 2 & 100 & 61 & 100 \\
\hline
\end{tabular}

Fuente: elaboración propia.

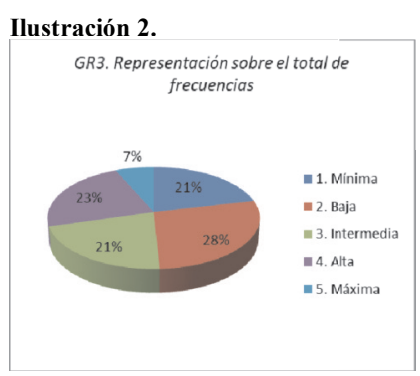

Fuente: elaboración propia.

En la tabla 3, se puede ver que el $28 \%$ de los participantes tiene un porcentaje de grasa corporal baja (2430 en mujeres y 16-21 en hombres); el 23\% tiene alta; el 21\% presenta un porcentaje de grasa mínima e intermedia; y tan solo un 7\% tiene el máximo porcentaje de grasa corporal. Véase en la ilustración 3. Si agrupamos estos datos en los valores positivos y negativos, se observa que el $49 \%$ de los sujetos presentan una grasa corporal saludable mientras que el $51 \%$ presenta unos valores negativos.

De forma general, no se encuentra diferencias significativas entre hombres y mujeres. No obstante, se observa que existe el mismo porcentaje de alumnas con un porcentaje grasa corporal tanto positiva como negativa (50\%). En cambio, un 54\% de alumnos presentan un porcentaje de grasa corporal negativa y un $46 \%$ presentan un valor positivo. 
En relación al rendimiento académico, no se observa diferencias significativas, debido a que existe el mismo porcentaje de personas (36\%) con un rendimiento académico bueno (notas entre 6 y 8), las cuales tienen una grasa corporal tanto positiva como negativa (37\%). En cuanto a los mejores resultados académicos (8-10) un $15 \%$ tienen un exceso de grasa corporal y un $13 \%$ un porcentaje considerado saludable, valores muy próximos.

\section{Porcentaje de Masa Muscular}

Considerando que para una mujer, el $30 \%$ y $40 \%$ de masa muscular es un valor positivo y saludable, y que para un hombre lo es en un 40\% y 50\% (Vera, 2021), se observa en la tabla 4 que todos los sujetos presentan valores saludables de masa muscular, entendiendo que los tres primeros intervalos (mínima, baja e intermedia) son aceptables y los dos últimos (alta y máxima) son óptimos.

Tabla 3.

Variable masa muscularen relación al sexo y al $R A$.

\begin{tabular}{|c|c|c|c|c|c|c|c|c|c|c|c|c|c|c|c|c|c|}
\hline & & & & \multicolumn{4}{|c|}{ SEXO } & \multicolumn{8}{|c|}{ RENDIMIENTO ACADÉMICO } & & \\
\hline & \multicolumn{3}{|c|}{ Tabla 4: Masa Muscular } & \multicolumn{2}{|c|}{ Femenino } & \multicolumn{2}{|c|}{ Masculino } & \multicolumn{2}{|c|}{$6-7}$. & \multicolumn{2}{|c|}{$7-8}$. & \multicolumn{2}{|c|}{ 8-9. } & \multicolumn{2}{|c|}{ 9-10. } & \multicolumn{2}{|c|}{ TOTAL } \\
\hline & & q & $\delta$ & $\mathrm{N}$ & $\%$ & $\mathrm{~N}$ & $\%$ & $\mathrm{~N}$ & $\%$ & $\mathrm{~N}$ & $\%$ & $\mathrm{~N}$ & $\%$ & $\mathrm{~N}$ & $\%$ & $\mathrm{~N}$ & $\%$ \\
\hline \multirow[b]{3}{*}{+} & 1. Mínima & $<34,78$ & $<56,06$ & 5 & 10 & 3 & 23 & 1 & 13 & 6 & 17 & 1 & 7 & 0 & 0 & 8 & 13 \\
\hline & 2. Baja & $34,79-38,56$ & $56,07-63,52$ & 14 & 29 & 5 & 38 & 4 & 50 & 9 & 25 & 5 & 33 & 1 & 50 & 19 & 31 \\
\hline & 3. Intermedia & $38,57-42,34$ & $63,53-70,98$ & 19 & 40 & 3 & 23 & 1 & 13 & 14 & 39 & 6 & 40 & 1 & 50 & 22 & 36 \\
\hline \multirow{3}{*}{++} & 4. Alta & $42,35-46,12$ & $70,99,78,44$ & 8 & 17 & 1 & 8 & 1 & 13 & 6 & 17 & 2 & 13 & 0 & 0 & 9 & 15 \\
\hline & 5. Máxima & $>46,13$ & $>78,45$ & 2 & 4 & 1 & 8 & 1 & 13 & 1 & 3 & 1 & 7 & 0 & 0 & 3 & 5 \\
\hline & \multicolumn{3}{|c|}{ Total general } & 48 & 100 & 13 & 100 & 8 & 100 & 36 & 100 & 15 & 100 & 2 & 100 & 61 & 100 \\
\hline
\end{tabular}

Fuente: elaboración propia.

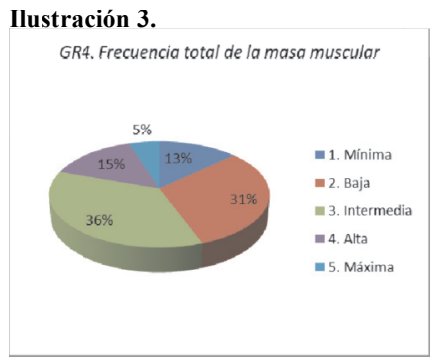

Fuente: elaboración propia.

Por lo tanto, se puede observar que un $80 \%$ dela muestra presenta una masa muscular aceptable y un $20 \%$ óptima. Asimismo, como se muestra la ilustración 4, la mayoría de los sujetos presentan una musculatura intermedia (36\%), donde la masa muscular para mujeres comprende entre 39-42 y para hombres entre 64-71; un 31\% tiene una masa muscular baja; un 15\% alta; un 13\% mínima; y tan solo un 5\% presenta la máxima masa muscular.

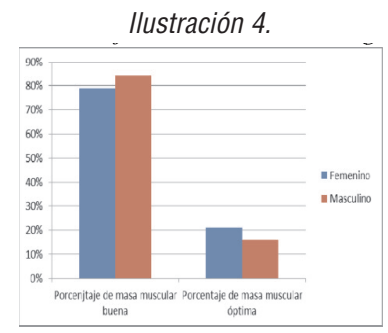

Fuente: elaboración propia. 


\section{LA COMPOSICIÓN CORPORAL COMO MEDICIÓN INDIRECTA DE LA ACTIVIDAD FÍSICA Y SU RELACIÓN CON EL RENDIMIENTO ACADÉMICO EN ESTUDIANTES DEL GRADO DE EDUCACIÓN INFANTIL}

\section{Porcentaje de masa muscular según el sexo}

En cuanto al sexo, no hay grandes diferencias significativas. Cabe destacar que ambos sexos presentan porcentajes superiores en cuanto a la masa muscular aceptable (mínima, baja e intermedia). Sin embargo, la mínima parte presentan niveles óptimos de masa muscular, con un $21 \%$ las mujeres y con un $16 \%$ los hombres.

En relación al rendimiento académico, se aprecia diferencias significativas, donde se observa que un total de 35 alumnos que tienen una masa muscular aceptable, es decir, el $57 \%$ de los sujetos, presentan un rendimiento académico bueno (notas entre 6-8) y un 23\% un rendimiento académico excelente (8-10). Por otro lado, tan solo un $5 \%$ de los alumnos que presentan una masa muscular óptima (alta y máxima) tienen los mejores resultados (entre 8 y 10).

\section{Densidad de Masa Ósea}

La cuarta variable a analizar es la masa ósea, la cual está íntimamente relacionada con el ejercicio, puesto que a consecuencia de éste, segrega la hormona osteocalcina, previniendo así la pérdida de memoria (Bouñoso, 2020). Según Miranda et al. (2015), una densidad ósea de >-1 es considerado normal; entre -1 y -2,5 se considera osteopenia; $\mathrm{y} \leq-2,5$ osteoporosis.

Tabla 4.

Variable Masa Ósea en relación al sexo y al RA.

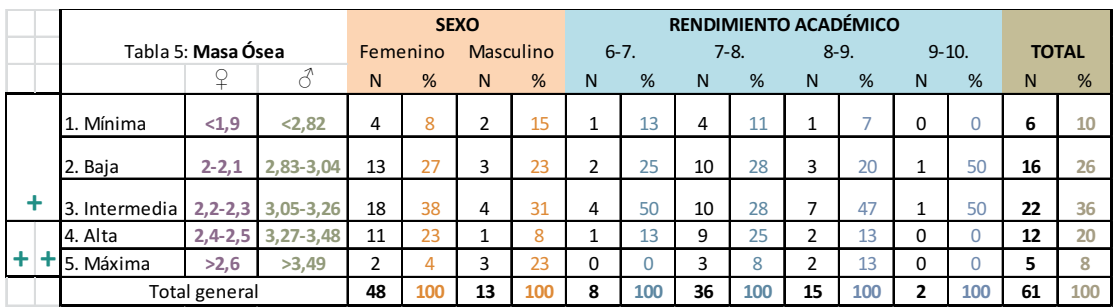

Fuente: elaboración propia.

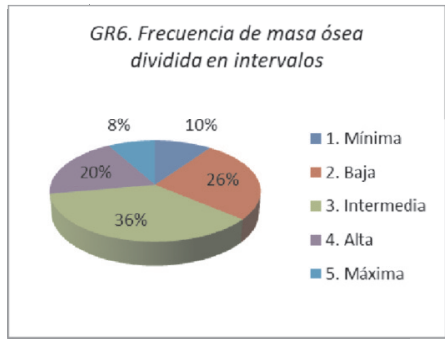

Fuente: elaboración provia.

Así pues, se puede observar en la tabla 5 , que toda la muestra presenta valores positivos y normales aunque se ha diferenciado a aquellos que presentan los valores buenos (mínima, baja e intermedia) con un $72 \%$ y aqueIlos con valores óptimos (altos y máximos) con un $28 \%$. Se puede comprobar en la ilustración 6 que, el $36 \%$ de los sujetos presentan una masa ósea intermedia (2,1-2,3 en mujeres y 3,04-3,26 en hombres), el 26\% baja, el $20 \%$ alta, el 10\% presenta el mínimo porcentaje de masa ósea y el 8\% el máximo.

Según la tabla añadida (tabla 5), el 73\% de las mujeres presentan una densidad ósea aceptable, mientras que el $27 \%$ una densidad ósea óptima. Por otro lado, sin observar grandes diferencias significativas, el $69 \%$ de 
Ios hombres presentan una densidad ósea aceptable y un 31\% óptima, valores muy próximos a la media total (72\% y $28 \%)$.

En cuanto a los mejores resultados académicos, las mejores calificaciones se encuentran en sujetos con un intervalo de masa ósea baja (50\%) e intermedia (50\%). Si agrupamos los datos, se observa que un total de 31 estudiantes (51\%) con una densidad de masa ósea aceptable presentan resultados académicos buenos, es decir, con notas comprendidas entre 6 y 8.

\section{Índice de Masa corporal (IMC)}

Caizàs (2020), establece a partir de los criterios SEED0, distintos grados para medir el IMC, donde: el peso insuficiente es considerado <18,5kg; un normopeso entre 18,5-24,9kg; sobrepeso de grado I entre 25-26,9; sobrepeso de grado II o preobesidad, comprendido entre 27-29,9; obesidad de grado I con valores entre 30-34,9; obesidad de tipo II entre 35-39,9; obesidad de tipo III (mórbida), comprendida entre 40-49,9; y, finalmente, la obseidad de tipo IV $\geq 50$. Se diferencia de los criterios de la OMS por tener unos intervalos más reducidos entre un criterio y otro, presentando así más grados de clasificación.

Tabla 5 .

Variable IMC en relación al sexo y al RA.

\begin{tabular}{|c|c|c|c|c|c|c|c|c|c|c|c|c|c|c|c|c|}
\hline & & & \multicolumn{4}{|c|}{ SEXO } & \multicolumn{8}{|c|}{ RENDIMIENTO ACADÉMICO } & & \\
\hline & & & \multicolumn{2}{|c|}{ Femenino } & \multicolumn{2}{|c|}{ Masculino } & \multicolumn{2}{|l|}{$6-7}$. & \multicolumn{2}{|l|}{ 7-8. } & \multicolumn{2}{|l|}{ 8-9. } & \multicolumn{2}{|l|}{ 9-10. } & \multicolumn{2}{|c|}{ TOTAL } \\
\hline & \multicolumn{2}{|c|}{ Tabla 6: Índice de Masa Corporal } & $\mathrm{N}$ & $\%$ & $\mathrm{~N}$ & $\%$ & $\mathrm{~N}$ & $\%$ & $\mathrm{~N}$ & $\%$ & $\mathrm{~N}$ & $\%$ & $\mathrm{~N}$ & $\%$ & $\mathrm{~N}$ & $\%$ \\
\hline \multirow[b]{2}{*}{+} & 1. Insuficiente & $<18,5$ & 3 & 6 & 0 & 0 & 0 & 0 & 2 & 6 & 1 & 7 & 0 & 0 & 3 & 5 \\
\hline & 2. Normopeso & $18,5-24,9$ & 32 & 67 & 6 & 46 & 5 & 63 & 23 & 64 & 9 & 60 & 1 & 50 & 38 & 62 \\
\hline \multirow{5}{*}{ - } & 3. Sobre peso grado I & $25-26,9$ & 5 & 10 & 3 & 23 & 1 & 13 & 5 & 14 & 1 & 7 & 1 & 50 & 8 & 13 \\
\hline & 4. Sobre peso grado II & $27-29,9$ & 3 & 6 & 3 & 23 & 2 & 25 & 2 & 6 & 2 & 13 & 0 & 0 & 6 & 10 \\
\hline & 5. Obesidad de tipo I & $30-34,9$ & 4 & 8 & 0 & 0 & 0 & 0 & 3 & 8 & 1 & 7 & 0 & 0 & 4 & 7 \\
\hline & 6. Obesidad de tipo II & $35-39,9$ & 1 & 2 & 1 & 8 & 0 & 0 & 1 & 3 & 1 & 7 & 0 & 0 & 2 & 3 \\
\hline & Total general & $40-49,9$ & 48 & 100 & 13 & 100 & 8 & 100 & 36 & 100 & 15 & 100 & 2 & 100 & 61 & 100 \\
\hline
\end{tabular}

Fuente: elaboración propia.

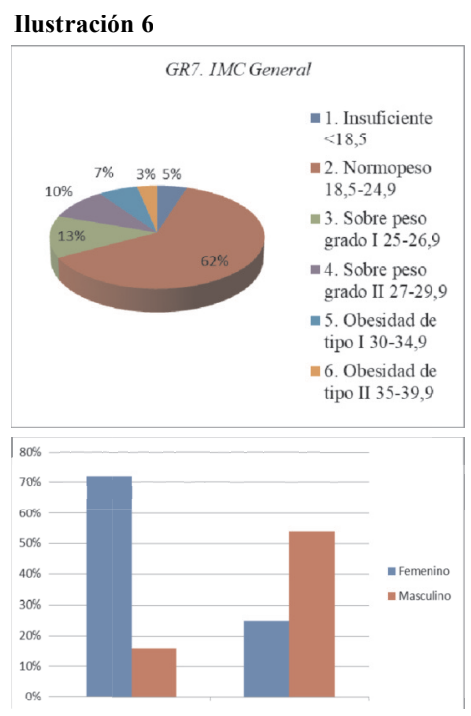




\section{LA COMPOSICIÓN CORPORAL COMO MEDICIÓN INDIRECTA DE LA ACTIVIDAD FÍSICA Y SU RELACIÓN CON EL RENDIMIENTO ACADÉMICO EN ESTUDIANTES DEL GRADO DE EDUCACIÓN INFANTIL}

Considerando que los intervalos 1 y 2 (insuficiente y normopeso) son valores positivos y el resto de los intervalos son un valor negativo de IMC, se puede decir que un $67 \%$ de los sujetos presentan un IMC saludable y un $33 \%$ no saludable, presentando desde un ligero sobrepeso hasta la obesidad.

El índice de masa corporal que nos indica la ilustración 7 , se observa que el $62 \%$ de los sujetos tienen un normopeso, es decir, un IMC comprendido entre 18,5 y 24,9; un 13\% presentan un sobrepeso de grado I; el 10\% sobrepeso de grado II; el 7\% tienen obesidad de tipo I; el $5 \%$ un IMC insuficiente; y finalmente, con $3 \%$ presenta obesidad de tipo II. Por lo que, se puede comprobar que más de la mitad de los participantes tienen normopeso.

En relación al sexo, cabe destacar que existen diferencias significativas. En la ilustración 8, se puede observar que el $72 \%$ de las mujeres presentan un IMC saludable, mientras que un $25 \%$ no. Sin embargo, el $54 \%$ de Ios hombres presentan un IMC negativo y tan solo un 16\% un IMC positivo. Por lo que, hay más hombres que sufren sobrepeso u obesidad y más mujeres que tienen un peso insuficiente o normopeso. En cuanto al rendimiento académico, en la tabla 6 se observa que un total de 30 personas que tienen un IMC positivo, es decir, un $49 \%$, presenta buenas notas, comprendidas entre 6-8 y un 18\% presenta el mejor rendimiento académico. Cabe destacar que las mejores calificaciones (9-10) se encuentran en personas con normopeso y sobrepeso de tipo I.

\section{Porcentaje de Agua Corporal}

Según James (2020), las mujeres suelen tener un porcentaje de agua menor que los hombres dado que tienden a tener más grasa y, efectivamente, en la tabla 7 se observa que las mujeres presentan menos porcentaje de agua que los hombres en cada uno de los intervalos calculados (mínimo, bajo, intermedio, alto y máximo). También expone que las mujeres suelen presentar entre un 52\%-55\% de agua corporal, mientras que los hombres un $60 \%$, por lo que, si agrupamos los intervalos en valores positivos y negativos, los tres primeros (mínimo, bajo e intermedio) son considerados intervalos con escaso porcentaje de agua corporal, mientras que los intervalos alto y máximo se consideran porcentajes de agua corporal aceptables y saludables. Así pues, se puede apreciar que el $59 \%$ de los estudiantes presentan niveles escasos agua corporal y el $41 \%$ tienen niveles de agua aceptable y saludable.

Tabla 6 .

Variable porcentaje de agua corporal en relación al sexo y al RA.

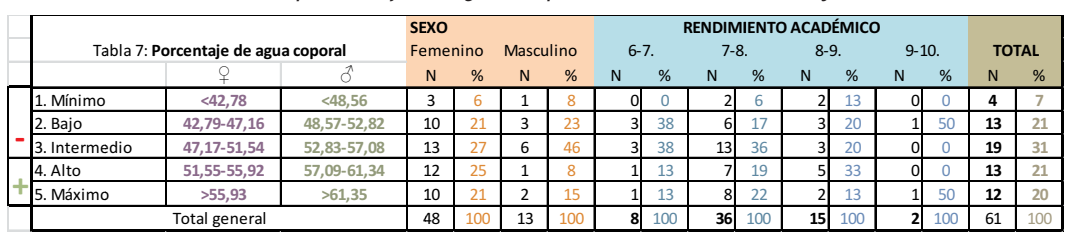

Fuente: elaboración propia.

Ilustración 8.

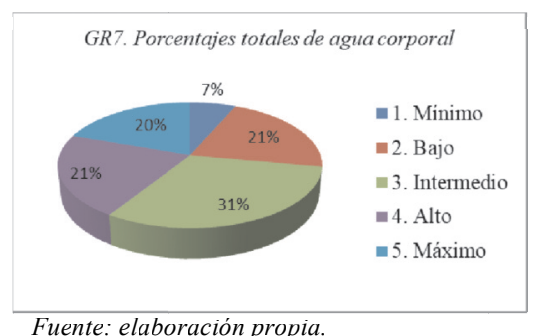

Fuente: elaboración propia. 
Según la tabla 7, un 31\% presentan niveles intermedios de agua corporal (47-52 en mujeres y 56-57 en hombres); un 21\% niveles bajos y altos de agua; con uno 20\% aquellos que presentan el máximo porcentaje de agua corporal; y con un 7\% aquellos que presentan la mínima agua corporal. Así pues, puede observarse en la ilustración 8 que la mínima parte de los sujetos presentan el mínimo nivel de agua corporal y el resto de los sujetos no presentan grandes diferencias debido a unos resultados similares.

En relación a la variable independiente del sexo, se observa que la mayoría de las mujeres y de los hombres presentan niveles escasos de agua corporal (valores negativos), las mujeres con un $54 \%$ y los hombres con un $77 \%$, encontrando mayores diferencias en hombres que en mujeres en relación a la media total (59\%).

En cuanto al rendimiento académico, la mayoría de los alumnos, con un $44 \%$ de los que presentan notas buenas comprendidas entre 6 y 8 , tienen un porcentaje de agua corporal escaso y solo aquellos que tienen un porcentaje de grasa corporal saludable, el 28\% tienen notas buenas y el 13\% notas óptimas.

\section{Grasa Visceral}

La grasa visceral es aquella que se encuentra en el interior de la cavidad abdominal, relacionándose con los problemas de salud. Las causas que aumenta la probabilidad de acumular grasa visceral es la edad, el sedentarismo y la alimentación. Ésta grasa se evalúa en una escala de 1 al 59 medida mediante la bioimpedancia, donde aquellos valores encontrados por debajo de 12 se consideran saludables y aquellos que lo superan se consideran peligrosos (García, 2021).

Tabla 7.

Variable grasa visceral en relación al sexo y al $R A$.

\begin{tabular}{|c|c|c|c|c|c|c|c|c|c|c|c|c|c|c|}
\hline \multirow[t]{3}{*}{ Tabla 8: Grasa Visceral } & \multirow{2}{*}{\multicolumn{4}{|c|}{$\begin{array}{l}\text { SEXO } \\
\text { Femenino Masculino }\end{array}$}} & \multicolumn{8}{|c|}{ RENDIMIENTO ACADÉMICO } & \multirow{2}{*}{\multicolumn{2}{|c|}{ TOTAL }} \\
\hline & & & & & \multicolumn{2}{|c|}{$6-7}$. & \multicolumn{2}{|c|}{$7-8}$. & \multicolumn{2}{|c|}{$8-9}$. & \multicolumn{2}{|c|}{$9-10}$. & & \\
\hline & $\mathrm{N}$ & $\%$ & $\mathrm{~N}$ & $\%$ & $\mathrm{~N}$ & $\%$ & $\mathrm{~N}$ & $\%$ & $\mathrm{~N}$ & $\%$ & $\mathrm{~N}$ & $\%$ & $N$ & $\%$ \\
\hline Saludable 1-12 & 48 & 100 & 12 & 92 & 8 & 100 & 36 & 100 & 14 & 93 & 2 & 100 & 60 & 98 \\
\hline \begin{tabular}{|l|} 
Peligroso $12-59$ \\
\end{tabular} & 0 & 0 & 1 & 8 & 0 & 0 & 0 & 0 & 1 & 7 & 0 & 0 & 1 & 2 \\
\hline Total general & 48 & 100 & 13 & 100 & 8 & 100 & 36 & 100 & 15 & 100 & 2 & 100 & 61 & 100 \\
\hline
\end{tabular}

Fuente: elaboración propia.

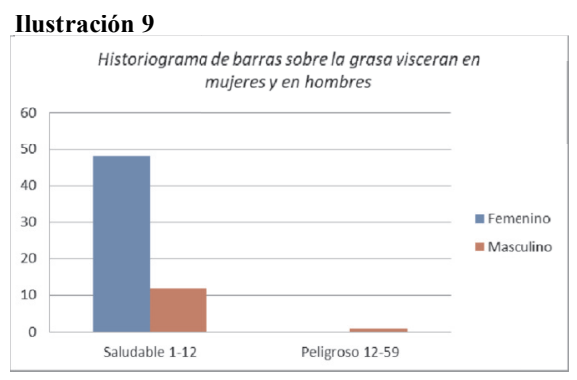

Así pues, como se observa en la tabla (tabla 8), casi el total de los sujetos presentan una grasa visceral saludables (98\%), mientras que un $2 \%$ presentan una grasa visceral peligrosa.

En relación al sexo, cabe destacar que todas las mujeres tienen una grasa visceral saludable, con un total de $100 \%$, mientras que los hombres con un $8 \%$ presentan niveles peligrosos de grasa visceral, cifra que supera a la media total (2\%).En relación con al resultado académico, si agrupamos los valores en rendimiento académico bueno (6-8) y rendimiento académico óptimo (8-10), se puede observar que 44 sujetos con grasa visceral saludable, es decir el $72 \%$ de estos, presentan notas buenas, mientras que el $26 \%$ presentan notas óptimas. 


\section{LA COMPOSICIÓN CORPORAL COMO MEDICIÓN INDIRECTA DE LA ACTIVIDAD FÍSICA Y SU RELACIÓN CON EL RENDIMIENTO ACADÉMICO EN ESTUDIANTES DEL GRADO DE EDUCACIÓN INFANTIL}

\section{Edad Metabólica}

Teniendo en cuenta que la media de edad cronológica es de 21 años, se considera que todos aquellos sujetos que estén por debajo del intervalo (21-24), son considerados valores positivos y aquellos que superen la media de 21 años cronológicos se consideran valores negativos. Así pues, en la tabla 9 se observa que el $61 \%$ de los sujetos presentan valores negativos y el $39 \%$ valores positivos, es decir, inferior a la media cronológica.

Tabla 8.

Variable edad metabólica en relación al sexo y al $R A$.

\begin{tabular}{|c|c|c|c|c|c|c|c|c|c|c|c|c|c|c|c|c|}
\hline & \multirow{3}{*}{\multicolumn{2}{|c|}{ Tabla 9: Edad Metabólica }} & \multicolumn{4}{|c|}{ SEXO } & \multicolumn{8}{|c|}{ RENDIMIENTO ACADÉMICO } & & \\
\hline & & & \multicolumn{2}{|c|}{ Femenino } & \multicolumn{2}{|c|}{ Masculino } & \multicolumn{2}{|c|}{ 6-7. } & \multicolumn{2}{|c|}{$7-8$} & \multicolumn{2}{|c|}{$8-9}$. & \multicolumn{2}{|c|}{$9-10}$. & \multicolumn{2}{|c|}{ TOTAL } \\
\hline & & & $\mathrm{N}$ & $\%$ & $\mathrm{~N}$ & $\%$ & $\mathrm{~N}$ & $\%$ & $\mathrm{~N}$ & $\%$ & $\mathrm{~N}$ & $\%$ & $\mathrm{~N}$ & $\%$ & $\mathrm{~N}$ & $\%$ \\
\hline \multirow[b]{4}{*}{+} & 1. & $12-15$ & 13 & 27 & 2 & 15 & 1 & 13 & 11 & 31 & 2 & 13 & 1 & 50 & 15 & 25 \\
\hline & 2. & $15-18$ & 1 & 2 & 0 & 0 & 0 & 0 & 0 & 0 & 1 & 7 & 0 & 0 & 1 & 2 \\
\hline & 3. & $18-21$ & 3 & 6 & 0 & 0 & 0 & 0 & 2 & 6 & 1 & 7 & 0 & 0 & 3 & 5 \\
\hline & 4. & 21-24 & 4 & 8 & 1 & 8 & 1 & 13 & 1 & 3 & 3 & 20 & 0 & 0 & 5 & 8 \\
\hline \multirow{6}{*}{-} & 5. & $24-27$ & 4 & 8 & 1 & 8 & 0 & 0 & 5 & 14 & 0 & 0 & 0 & 0 & 5 & 8 \\
\hline & 6. & $27-30$ & 0 & 0 & 1 & 8 & 1 & 13 & 0 & 0 & 0 & 0 & 0 & 0 & 1 & 2 \\
\hline & 7. & $30-33$ & 3 & 6 & 2 & 15 & 1 & 13 & 4 & 11 & 0 & 0 & 0 & 0 & 5 & 8 \\
\hline & \begin{tabular}{|l|}
8. \\
\end{tabular} & $33-36$ & 16 & 33 & 5 & 38 & 3 & 38 & 11 & 31 & 6 & 40 & 1 & 50 & 21 & 34 \\
\hline & 9. & 36-39 & 4 & 8 & 1 & 8 & 1 & 13 & 2 & 6 & 2 & 13 & 0 & 0 & 5 & 8 \\
\hline & \multicolumn{2}{|c|}{ Total general } & 48 & 100 & 13 & 100 & 8 & 100 & 36 & 100 & 15 & 100 & 2 & 100 & 61 & 100 \\
\hline
\end{tabular}

Fuente: elaboración propia.

\section{Ilustración 10.}

GR9. Frecuencia de edad metabólica

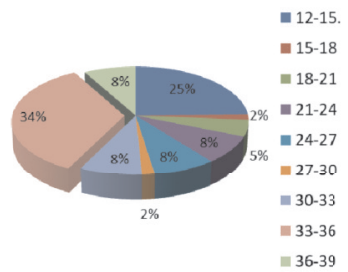

\section{Ilustración 11}

GR10. Frecuencia en el nivel de AF según las respuestas de los sujetos

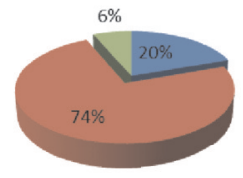

1. Poco o nada

2. En ocasiones y de baja intensidad

11. $10 \mathrm{~h}$ semanalaes $y$ de alta intensidad

Fuente: elaboración propia.

Como se observa en la ilustración 9, la edad metabólica más común es de 33-36 años, con un 34\%; por debajo, con un $25 \%$ nos encontramos aquellos que tienen una edad metabólica opuesta a la anterior (12-15 años); un 8\% presentan edades metabólicas comprendidas entre 21-24, 24-27, 30-33 y 36-39 años; un 5\% tienen una edad metabólica de 18-21; y finalmente, un 2\% tienen entre 15-18 y 27-30 años metabólicos. Así pues, se observa claramente que más de una cuarta parte tienen una edad metabólica de 33-36, seguida de 12-15 años.

En relación al género, si agrupamos los valores en positivos y negativos, se observa diferencias significativas en hombres, debido a que un $77 \%$ presentan valores negativos (edad metabólica superior a 24) y un $23 \%$ con valores positivos. No obstante, se encuentra porcentajes similares entre mujeres con valores tanto negativos (53\%) como positivos (43\%).

En cuanto al rendimiento académico, se observa que un total de 28 alumnos y alumnas, es decir el $46 \%$ de la muestra, con edades cronológicas superiores a la media, presentan un rendimiento académico bueno, entre 6 y 8 . Pero solo el $13 \%$ de aquellos que tienen valores positivos en cuanto a la edad metabólica, presentan resultados académicos óptimos. 


\section{Nivel De Actividad Física}

Como se ha mencionado anteriormente, se pretende analizar también la sencilla pregunta que tuvieron que responder para que la báscula de bioimpedancia calculara los resultados. Así pues, se observa en la ilustración 10, que el 74\% de los participantes respondieron que en ocasiones realizan actividad física y de baja intensidad; el 20\% respondió que realizaban poca actividad física o nada; y un 7\% realizaban 10 horas semanales de actividad física y de alta intensidad.

Tabla 9.

Nivel de actividad física en relación al sexo y al RA.

\begin{tabular}{|c|c|c|c|c|c|c|c|c|c|c|c|c|c|c|c|}
\hline & \multirow[b]{3}{*}{ Tabla 10: Nivel de Actividad Física } & \multicolumn{4}{|c|}{ SEXO } & \multicolumn{8}{|c|}{ RENDIMIENTO ACADÉMICO } & \multirow{2}{*}{\multicolumn{2}{|c|}{ TOTAL }} \\
\hline & & \multicolumn{2}{|c|}{ Femenino } & \multicolumn{2}{|c|}{ Masculino } & \multicolumn{2}{|c|}{ 6-7. } & \multicolumn{2}{|c|}{ 7-8. } & \multicolumn{2}{|c|}{$8-9}$. & \multicolumn{2}{|c|}{ 9-10. } & & \\
\hline & & $\mathrm{N}$ & $\%$ & $\mathrm{~N}$ & $\%$ & $\mathrm{~N}$ & $\%$ & $\mathrm{~N}$ & $\%$ & $\mathrm{~N}$ & $\%$ & $\mathrm{~N}$ & $\%$ & $\mathrm{~N}$ & $\%$ \\
\hline$=$ & 1. Poco o nada & 12 & 25 & 0 & 0 & 1 & 13 & 5 & 14 & 6 & 40 & 0 & 0 & 12 & 20 \\
\hline & 2. En ocasiones y de baja intensidad & 34 & 71 & 11 & 85 & 6 & 75 & 28 & 78 & 9 & 60 & 2 & 100 & 45 & 74 \\
\hline+ & 3. $10 \mathrm{~h}$ semanalaes y de alta intensidad & 2 & 4 & 2 & 15 & 1 & 13 & 3 & 8 & 0 & 0 & 0 & 0 & 4 & 7 \\
\hline & Total general & 48 & 100 & 13 & 100 & 8 & 100 & 36 & 100 & 15 & 100 & 2 & 100 & 61 & 100 \\
\hline
\end{tabular}

Fuente: elaboración propia.

Si agrupamos los valores en positivos, donde las personas sí realizan actividad física adecuada y en negativos, donde se considera que las personas realizan una insuficiente actividad física, se observa en la tabla 10 que, un $80 \%$ de los sujetos realizan una actividad física adecuada en su día a día, mientas que un $20 \%$ presenta una actividad física insuficiente. En relación al sexo, ningún chico respondió que realizara poca o ninguna actividad física (0\%), por lo que todos los chicos realizan AF ya sea de baja intensidad como de alta. En cambio, un 25\% de las mujeres respondió que no hacían actividad física o hacían poca y un 75\% respondieron que sí hacían ejercicio, tanto de baja intensidad como de alta. Por otro lado, si agrupamos los resultados académicos en buenos (comprendido entre 6 y 8 ) y óptimos (comprendido entre 8 y 10), se observa que 38 sujetos que realizan una AF adecuada, es decir el $62 \%$ de la muestra, presentan un rendimiento académico bueno (6-8), mientras que el $18 \%$ presenta un rendimiento académico óptimo (8-10).

\section{DISCUSIÓN}

En base a los resultados obtenidos, se puede decir que los estudiantes del 3ํㅓㅇ del Grado de Educación infantil presentan niveles saludables en cuanto a la variable de peso. No obstante, teniendo en cuenta que la variable de peso proporciona escasa información, se interpreta que son personas con hábitos saludables y que realizan un nivel adecuado de actividad física. Asimismo, cabe destacar que una cuarta parte obtienen unos resultados académicos óptimos.

En cambio, el porcentaje de grasa corporal sí proporciona mayor información, ya que se relaciona con un mejor hábito de vida saludable relacionada a una vida activa, donde autores como Cardozo, et al. (2016), afirma que un elevado porcentaje de grasa corporal está asociada a diversos factores de enfermedades cardiovasculares y evidencia un progresivo aumento de sobrepeso en estudiantes universitarios. Estas afirmaciones están en línea con los resultados obtenidos en el presente estudio puesto que, la mitad de la muestra presenta niveles saludables en cuanto al porcentaje de grasa corporal y la otra mitad niveles no tan saludables, sin encontrarse realmente diferencias significativas. Según Ortega (2020), existe una asociación clara entre el porcentaje de grasa corporal y el rendimiento académico. Esta afirmación no puede ser discutida puesto que, no se puede sacar una interpretación clara sobre el rendimiento académico debido a la diferencia de los resultados obtenidos, ya que las mejores calificaciones están presentes en sujetos con una grasa corporal mínima pero también alta.

En cuanto a los porcentajes de masa muscular, si tenemos en consideración los valores que expone Vera (2021) en su estudio, donde los porcentajes de masa muscular saludables en mujeres comprende entre un 30$40 \%$ y en hombres entre $40-50 \%$, se puede decir que los estudiantes del $3 \%$ curso del grado de Educación Infantil 


\section{LA COMPOSICIÓN CORPORAL COMO MEDICIÓN INDIRECTA DE LA ACTIVIDAD FÍSICA Y SU RELACIÓN CON EL RENDIMIENTO ACADÉMICO EN ESTUDIANTES DEL GRADO DE EDUCACIÓN INFANTIL}

presentan niveles adecuados de masa muscular. Una pequeña parte presenta nieles superiores a los mencionados, por lo que se puede interpretar que en vista al porcentaje de masa muscular, los estudiantes tienen unos hábitos de vida saludable en cuanto a la actividad física. Por otro lado, se ha observado que la mayoría de las personas que tienen una masa muscular aceptable presentan un rendimiento académico bueno y en menor medida un rendimiento académico excelente.

En la variable de masa ósea, si se considera que los parámetros de densidad ósea normales y saludables son >-1 (Miranda, et al., 2013), se demuestra que los estudiantes presentan valores muy positivos. Como bien dice Escalante y Franco-Vicario (2003), la reducción ósea se relaciona con el sedentarismo. No obstante, de acuerdo con Bouñoso (2020), el cual menciona que la densidad de masa ósea está íntimamente relacionada con el ejercicio, se interpreta que los estudiantes presentan buenos niveles de actividad física, siendo personas activas, sanas y sin riesgo de sufrir osteopenia ni osteoporosis.

Los niveles de IMC que se han utilizado para el estudio están basados en los criterios SEEDO que la sociedad Española de Obesidad (2020) ofrece para definir la obesidad en grados (Caixàs, 2020). Así pues, se puede afirmar que gran mayoría de los estudiantes presentan niveles saludables de IMC, siendo más común en mujeres que en hombres, debido a que la mayoría de los hombres presentan un IMC negativo, considerado sobrepeso u obesidad. Por otro lado, las mejores calificaciones se encuentran en personas con normopeso y sobrepeso de tipo I, afirmando así las conclusiones de Barth et.al (2021), quien demuestra que no existe asociaciones significativas entre la AF y el RA en su estudio y de Tarp et al. (2021), quien demuestra que el aumento de horas de educación física no mejora el RA de los alumnos en relación con el horario estándar.

En línea con las afirmaciones de James (2020), los resultados demuestran que las mujeres suelen tener un porcentaje de agua menor que los hombres. Sin embargo, se observa que casi la mitad de los estudiantes presentan niveles escasos de agua corporal, siendo algo superiores que aquellos que presentan niveles adecuados y saludables de agua. En este sentido, se interpreta que los estudiantes tienen hábitos no muy saludables en relación a la hidratación debido a que suelen descuidar la hidratación tras practicar actividad física (Soteras, 2014). Por ello, se considera que los estudiantes realizan un adecuado nivel de actividad física pero con malos hábitos de hidratación. Por otro lado, aquellos alumnos y alumnas que presentan las mejores calificaciones son las que tienen los niveles más bajos de agua, es decir, aquellos que realizan actividad física.

De acuerdo con los valores saludables y peligrosos que expone García (2021) en relación a la grasa visceral, casi la totalidad de los estudiantes presentan niveles adecuados y saludables, mientras que una muy pequeña parte tiene una grasa visceral peligrosa. Asimismo, se considera que los estudiantes tienen un hábito de vida saludable, con una alimentación sana y una vida activa. Los resultados están en línea con el estudio realizado por Wunsch et al. (2021), quien afirma la relación positiva entre rendimiento académico y actividad física, debido a que la gran mayoría presenta niveles positivos de grasa visceral y un buen rendimiento académico.

En cuanto a la edad metabólica obtenida en los resultados, se puede decir que la mayoría de los estudiantes muestran edades superiores a la media de edad cronológica (21 años), por lo que el nivel de salud que describe su estado físico y actividad es inferior a los que corresponde con su edad cronológica. La edad metabólica más frecuente queda comprendida entre 33-36 años. Esto quiere decir que, existen hábitos no saludables en su día a día, por ejemplo, una escasa actividad física.

En relación a la pregunta que respondieron los estudiantes para que la báscula de bioimpedancia calculara Ios resultados, se confirma que los estudiantes realizan actividad física, ya sea alta intensidad o de baja intensidad, siendo ésta última la más común. Una muy pequeña parte reconoce que no hace actividad física. Dado que todos presentan un buen rendimiento académico, los resultados coinciden con las conclusiones obtenidas por Conde y Tercedor (2014), quienes afirman la existencia de una relación positiva, concluyendo que los niveles de AF favorecen el RA de los adolescentes y universitarios. 


\section{CONCLUSIONES}

En primer lugar, con este trabajo se ha alcanzado el objetivo principal puesto que, mediante un análisis descriptivo, se ha conseguido conocer indirectamente el nivel de actividad física y hábitos saludables mediante el análisis de la composición corporal de los estudiantes del aula de $3^{0}$ del grado en Educación Infantil, así como la relación existente entre sexos y rendimiento académico. Los elementos de la composición corporal como el peso, el porcentaje de masa muscular, la masa ósea, el IMC en mujeres, el porcentaje de agua corporal y la grasa visceral, nos indica que sí realizan actividad física. En cambio, el porcentaje de masa corporal, el IMC en hombres y la edad metabólica son aspectos contradictorios, ya que nos indica una actividad física insuficiente en los sujetos.

No obstante, a modo de conclusión, se afirma que los estudiantes del $3^{0}$ curso del grado en Educación Infantil presentan un buen estado de condición física y de salud, de manera que, se llega a la conclusión de que realizan un nivel adecuado de actividad física. Sin embargo, todos los estudiantes presentan buenos resultados académicos presentando poca variabilidad entre las notas, por este motivo, no se han podido sacar conclusiones claras sobre la actividad física en relación al rendimiento académico. Si bien, los resultados del presente estudio, sugieren la realización de actividad física como forma de vida saludable para encontrar mejoras en el rendimiento académico.

Por otro lado, las limitaciones de estudio encontradas han sido: tener una muestra pequeña, la homogeneidad de la muestra en cuanto al sexo y la homogeneidad en cuanto a las notas académicas. Pese a todo, se debe resaltar la metodología realizada para describir a un grupo de estudiantes. Así pues, para futuras investigaciones, se propone realizar un estudio similar con una muestra mayor y heterogénea, donde se puedan sacar conclusiones más claras en cuanto a la influencia de la actividad física en el rendimiento académico. También se propone analizar los hábitos de vida y satisfacción personal de las personas adultas midiendo su composición corporal.

Para terminar, me gustaría destacar lo enriquecedor que me ha resultado este trabajo en cuanto a los conocimientos en investigación. He descubierto lo beneficioso que es la producción científica para el conocimiento de la realidad actual como forma de detectar problemas para crear soluciones.

\section{REFERENCIAS BIBLIOGRÁFICAS}

Alfaro, A. A., Santiago, C. y Cortés, M. E. (2018). Innovación didáctica en el aula de Biología: cerebro activo en un cuerpo activo. UBO Health Journal, 5(2), 26-37. https://cutt.ly/dnp66bp

Barrios, L. y López, M.A. (2016). Aportes del ejercicio físico a la actividad cerebral. Educación Física y Deportes, Revista Digital. 16. 1-7. https://cutt.ly/UnaqqYk

Barth, I., Ragnvald, K., Alfred, S., Eikeland, H. \& Thurston, M. (2021). Physical activity and academic achievement among Norwegain adolescents: Findings from a longitudinal study. Preventive Medicine Reports, 21(1), 17. https://doi.org/10.1016/j.pmedr.2021.101312.

Bouñoso, M. (2020). Ejercicio y memoria. https://cutt.ly/nmKOhH7

Caixàs, A., Villaró, M., Arraiza, C., Montalvá, J. C., Lecube, A., Fernández-García, J. M., ... \& Tinahones, F. J. (2020). Documento de consenso de la Sociedad Española de Obesidad (SEEDO) y de la Sociedad Española de Médicos de Atención Primaria (SEMERGEN) sobre la continuidad asistencial en obesidad entre Atención Primaria y Unidades Especializadas Hospitalarias 2019. Medicina Clínica, 155(6), 267-e1. Doi: https://doi.org/gfq2

Cardozo, L. A., Cuervo, Y. y Murcia, J. (2016). Porcentaje de grasa corporal y prevalencia de sobrepeso-obesidad en estudiantes universitarios de rendimiento deportivo de Bogotá, Colombia. Nutrición clínica y dietética hospitalaria, 36(3), 68-75. https://cutt.ly/qnp684Z

Cid, F. M. (2016). Plasticidad sináptica, BDNF y ejercicio físico. EmásF: revista digital de educación física, (40), 51-63. https://dialnet.unirioja.es/descarga/articulo/5456613.pdf

Conde, M.A. y Tercedor, P. (2014). La actividad física, la educación física y la condición física pueden estar rela- 


\section{LA COMPOSICIÓN CORPORAL COMO MEDICIÓN INDIRECTA DE LA ACTIVIDAD FÍSICA Y SU RELACIÓN CON EL RENDIMIENTO ACADÉMICO EN ESTUDIANTES DEL GRADO DE EDUCACIÓN INFANTIL}

cionadas con el rendimiento académico y cognitivo en jóvenes. Revisión sistemática. Archivos de medicina del deporte, 32(2), 100-109. https://cutt.ly/DmKOlhT

Cortés, M. E., Veloso, B. C. y Alfaro, A. A. (2020). Impacto de la actividad física en el desarrollo cerebral y el aprendizaje durante la infancia y la adolescencia. Infancia, Educación y Aprendizaje (IEYA), 7(1), 39-52. https://cutt.ly/hnp623h

Dávila-Batista, V., Gómez-Ambrosi, J., Fernández-Villa, T., Molina, A. J., Frühbeck, G. y Martín, V. (2016). Escala colorimétrica del porcentaje de grasa corporal según el estimador de adiposidad CUN-BAE. Atención Primaria, 48(6), 422. Doi: https://doi.org/gfqs

Escalante, M., \& Franco-Vicario, R. (2003). Deporte y masa ósea. Revista Española de Enfermedades Metabólicas Óseas, 12(4), 80-82. https://medes.com/publication/11224

García, Y. (2021). Grasa Visceral: Conoce el indice de grasa visceral y su nivel ideal. https://cutt.ly/Mnaqdrw

Grasa vs. Músculo: diferencias, pesos y porcentajes (2020) https://cutt.ly/qmKOxIE

James, L. (2020). Introducción al agua corporal. https://cutt.ly/onp5BJZ

Lara, J., Siervo, M., Bertoli, S., Mathers, J.C., Battezati, A., Ferraris, C. \& Tagliabue, A. (2014). Accuracy of the novel predictive methods for measurements os fat mass in healthy older subjects. Aging clinical and experimental research, 26(3). https://cutt.ly/amKOve9

Manual de uso - Tanita BC-601F Báscula. https://manuall.es/tanita-bc-601f-bascula/

Miranda, V. E., Muñoz, C. S., Paolinelli, G. P. y Astudillo, A. C. (2013). Densitometría ósea. Revista Médica Clínica Las Condes, 24(1), 169-173. Doi: https://doi.org/f2x593

Ortega, M. Á. (2020). Condición física, composición corporal y rendimiento académico en niños/as con sobrepeso/obesidad. SPORT TK-Revista EuroAmericana de Ciencias del Deporte, 9(2), 47-56. https://doi.org/gfqv

Ponce, H. F. (2015). Influencia de un Programa de Actividad Física sobre los procesos cognitivos de las personas mayores de 60 años (Tesis doctoral). Universidad de Granada, Andalucía, España.

Santos, M.G. (2011). Aplicación de nuevas tecnologías al análisis de la composición corporal: Contraste metodológico y utilidad en el diagnóstico de la condición nutricional (tesis doctoral). Universidad Complutense de Madrid, Madrid, España.

Soteras, A. (2014). La hidratación del deportista: agua pero también ... bebidas isotónicas. https://cutt.ly/hmKObQ0

Tarp. J., Gejl, A.K., Hillman, C.H. Wedderkopp, N. y Bugge, A. (2021). Does Additional Physical Education Improve Exam Performance at the End of Compulsory Education? A Secondary Analysis from a Natural Experiment: The CHAMPS-Study DK. Children, 8(57), 1-11. Doi: https://doi.org/gfjk

Wunsch, K., Fiedler, J., Beacher, B. \& Woll, A. (2021). The Tridirectional Relationship among Physical Activity, Stress, and Academic Performance in University Students: A Systematic Review and Meta-Analysis. Environmental Research and Public Health, 18(739), 1-18. Doi: https://doi.org/gfjh 